\title{
Examining the sensitivity of the surveillance system to detect TB cases in Afghanistan
}

Naqibullah Hamdard ( $D$ Hamdardnaqibullah@gmail.com )

MoPH https://orcid.org/0000-0003-2381-1220

\author{
Alim Atarud \\ Khalid Seddiq \\ NTP \\ Anwar Hanif \\ Care International
}

United Nations Development Programme

Research article

Keywords: TB, Surveillance, Sensitivity, Active Case Findings, PHC

Posted Date: August 20th, 2020

DOI: https://doi.org/10.21203/rs.3.rs-46734/v1

License: (9) This work is licensed under a Creative Commons Attribution 4.0 International License.

Read Full License 


\section{Abstract}

\section{Background}

Tuberculosis (TB) remains a global public health threat. World Health Organization (WHO) End TB strategy recommends that effective TB control relies on general health systems, especially, on integrated and well-functioning PHC facilities. Despite, integration in Basic Package of Health Services (BPHS), evidence demonstrates that a large number of TB cases are not captured. 25000 TB cases are missed every year (Aloudal, 2015). 49\% of individuals, recorded at health facilities as presumptive to have TB have not been screened (HMIS, 2016). This study intended to evaluate different dimensions of TB surveillance system and the Primary Health Care (PHC) facilities' role in TB active case finding.

\section{Methods}

We conducted a cross-sectional study. The study was implemented in eleven provinces of Afghanistan in from August to November 2016. The geographic and demographic representativeness determined the choice of provinces. All primary health care facilities in studied provinces equated to 870 , therefore, considering a $95 \%$ confidence interval, a sample size of 161 facilities established the sample frame. A stratified sampling strategy facilitated the selection of sampled facilities within different categories from an inclusive list of all facilities.

\section{Results}

The study found that the overall sensitivity of TB surveillance systems in-country is $56.30 \%$. This means that $43.70 \%$ of 171 TB cases had remained undetected. $43.81 \%$ of studies facilities used at least one accepted active case finding strategy while $56.20 \%$ implemented none. In studied areas, $83.33 \%$ of 11 studied DHs and $69.57 \%$ of 35 studied CHCs had a referral system for MDR-TB patients.

\section{Conclusion}

To enhance sensitivity and boost case findings, it is essential to implement case-finding strategies targeted at high-risk groups in specific areas. The high-risk groups include IDPs, returnees, slum residents, prisoners, and addicts. Additionally, it is necessary to train private pharmacists and traditional healers to identify and refer individuals with TB symptoms for follow up and further evaluation at the PHC level.

\section{Background}

Tuberculosis (TB) remains a global public health threat. In 2015, each year, 10 million people become ill with TB. However, TB is curable 1.5 million people die of TB every year and this makes world's first infectious killer (WHO, 2020). WHO End TB Strategy envisions a 95\% reduction in TB deaths and an $80 \%$ decrease in TB incidence by 2030 (WHO, 2016). In Afghanistan, the decline rate in TB incidence has been reported at 1.5\% since 2014 (WHO, 2016) but the deliberate intended reduction requires a higher decline 
rate of $5 \%$. The strategy concedes that achieving the milestone relies on the strength of the surveillance system and the active case finding strategies in the vulnerable populations.

TB is still a big public health problem in Afghanistan. 65000 cases and 11000 deaths were estimated to be caused by TB in 2017 (WHO, 2020).

The National Tuberculosis Control Program (NTP) provides leadership, coordination, capacity development on aspects related to TB care. NTP delivers lab reagents, anti TB medicines, and DOTS training.

NTP is a government program that works under the authority of MOPH and directly reports to the Communicable Disease Control (CDC) Directorate. NTP has a TB task force in place to coordinate the activities of all stakeholders and to implement MOPH/NTP policies, strategies, and standards. All the strategic decisions are made centrally at the Kabul Head Office. NTP has 34 provincial offices in each province of Afghanistan and they are responsible for the implementation, monitoring, and supervision of the TB Programme (Ernst \& Young, 2015).

Despite, integration in the Basic Package of Health Services (BPHS), evidence demonstrates that many TB cases are not captured by PHC. 25000 TB cases are missed every year (Aloudal, 2015). The National Health Management Information System (HMIS) highlights that $49 \%$ of individuals, recorded at primary health facilities as presumptive to have TB have not been screened.

Other matters related to low TB cases detection include lack of robust case-finding strategies, the low capacity of health workers, insufficient lab efficiency, inadequate TB supply, and insufficient patients' education (Seddiq, 2014).

This study intends to examine the different dimensions of the surveillance system for TB case detection and evaluate the level of PHC facilities involvement in TB active case finding.

\section{Methodology}

The methodology section includes the sampling strategy, data collection, study instruments, the analysis plan, and ethical considerations.

Sampling strategy

This is a cross-sectional study; implemented in eleven provinces of Afghanistan. The geographic and demographic representativeness determined the choice of provinces. The selected provinces comprised Kabul, Herat, Nangarhar, Kandahar, Balkh, Badakhshan, Bamyan, Farah, Khost, Kunar, and Samangan. Traditionally, Afghanistan is divided into different demographic and geographical zones ( south, southeast, southwest, east, west, north, northeast, and central), selected provinces represent different zones. 
As all PHC facilities in studied provinces equated to 870 , therefore, considering a $95 \%$ confidence interval, a sample size of 161 facilities established the sample frame. A stratified sampling strategy facilitated the selection of sampled facilities within different categories from an inclusive list of facilities.

Each category of health facilities in the list was deliberated as a stratum. The categories contained District Hospital (DH), Comprehensive Health Center (CHC), Basic Health Center (BHC), Sub Health Center (SHC), and Mobile Health Team (MHT). The number of sampled facilities in each stratum was proportionate to the number of all health facilities in that stratum. The stratum specific sample sizes were determined using following equation: $n_{h}=(\mathrm{NH} / \mathrm{N}) * n$

Equation 1 Stratified sampling

Where " $\mathrm{n}_{\mathrm{h}}$ " is the sample size of the stratum. "Nh" is the population size of the stratum. "N" is the total population size and " $n$ " is the total sample size.

Data collection, monitoring, and supervision

We trained provincial NTP officers to collect the data. We also prepared the national NTP officers to undertake supervisions in assigned provinces during the data collection phase. The national TB officers provided technical support to enumerators and verified the sites, the enumerators had visited.

We utilized the following tools for data collections:

Observation checklist

The observation checklist included managerial, administrative, patient management, physical environment, and case finding sections.

Interview instruments

The semi-structured tool included questions on active case finding strategies, guidelines, checklists, and indicators.

Data management and quality assurance

We employed a five-step approach to ensure the quality of the study data. We primarily designed the study tools with complementarity in mind, we strategized to have some core similar questions to ask from different respondents to enable triangulation, secondly, we required all enumerators to get filled questionnaires and observation checklists signed by health facility managers, thirdly, we called different facilities to verify enumerators had visited intended facilities, fourthly, we patterned all filled questionnaires for completeness and accuracy, finally, we crossed matched the entered data with hard copies. Additionally, we integrated the following measures in all phases of this study.

1. Deploying the high-quality study team. 
2. Training of provincial NTP officers for data collection as they were acquainted with the work.

3. Enabling national NTP officers to supervise the data collection process at the provincial and health facility level.

4. Translating study tools to local languages. The translated tools were retranslated to English by a third party to ensure the integrity of questions and concepts.

5. Training of enumerators

6. The pilot of study instruments

7. The entry of collected data into password protect databases with established rules to limit the volume of missed data.

8. Extensive monitoring and supervision of data collection, entry, and analysis.

\section{Data analysis}

The sensitivity of surveillance and information system to detect TB cases

We calculated the proportion of TB cases detected by the TB surveillance system and the proportion of those cases in the community. To calculate the sensitivity, we used the health events by the gold standards table (CDC, 2016). In principle, gold standards are regarded as the best screening tests or procedures under reasonable conditions. Here, we measure the accuracy of TB surveillance in Afghanistan by comparing it against the gold standard test in a $2 \times 2$ table to calculate its sensitivity and precision. CDC updated surveillance evaluation guideline also uses a $2 \times 2$ Table 1 to measure surveillance system sensitivity.

Table 1

$2 \times 2$ tables of calculating sensitivity

\begin{tabular}{|lllll|}
\hline \multirow{2}{*}{ Detected by surveillance } & \multicolumn{4}{l}{ Yealth events by gold standards } \\
\cline { 2 - 5 } & Yes & A (True positive) & B (False positive) & A + B \\
\hline No & C ( False negative) & D ( True negative) & C + D \\
\hline & A + C & B + D & \\
\hline
\end{tabular}


Table 2

Primary health care system sensitivity to detect TB cases (There is a limitation that province-specific TB prevalence is not available).

\begin{tabular}{|c|c|c|c|c|c|}
\hline Provinces & $\begin{array}{l}\text { Provincial } \\
\text { Population } \\
\text { (2015- } \\
2016)\end{array}$ & $\begin{array}{l}\text { TB } \\
\text { prevalence } \\
\text { rate per } \\
100000\end{array}$ & $\begin{array}{l}\text { Estimated } \\
\text { number of } \\
\text { TB cases in } \\
\text { each } \\
\text { province }\end{array}$ & $\begin{array}{l}\text { All TB positive cases } \\
\text { from the annual } \\
\text { report on cases } \\
\text { registration NTP, } \\
2016\end{array}$ & $\begin{array}{l}\text { The sensitivity of } \\
\text { the primary health } \\
\text { care system } \\
\text { detected TB cases }\end{array}$ \\
\hline Kabul & $4,372,977$ & 340 & 14868 & 6108 & $41.08 \%$ \\
\hline Herat & $1,890,202$ & 340 & 6427 & 3194 & $49.70 \%$ \\
\hline Nanrhar & $1,517,388$ & 340 & 5159 & 4530 & $87.81 \%$ \\
\hline Balkh & $1,325,659$ & 340 & 4507 & 2169 & $48.12 \%$ \\
\hline Kandahar & $1,226,593$ & 340 & 4170 & 2626 & $62.97 \%$ \\
\hline Badakhsh & 950,953 & 340 & 3233 & 1076 & $33.28 \%$ \\
\hline Khost & 574,582 & 340 & 1954 & 1985 & $101.61 \%$ \\
\hline Kunar & 450,652 & 340 & 1532 & 1184 & $77.27 \%$ \\
\hline Bamyan & 447,218 & 340 & 1521 & 455 & $29.92 \%$ \\
\hline Samangan & 387,928 & 340 & 1319 & 581 & $44.05 \%$ \\
\hline Farah & 507,405 & 340 & 1725 & 750 & $43.47 \%$ \\
\hline Total & $13,651,557$ & 340 & 46415 & 24658 & $56.30 \%$ \\
\hline
\end{tabular}

Ethical Consideration

The study received ethical approval from MoPH's Institutional Review Board (IRB). All participants provided informed consent before participating in the study. For patients, the interviewers read the informed consent and described the objectives, benefits, voluntary participation, confidentiality, and rights of respondents' withdrawal. The enumerators had also got trained on local cultural norms and socially acceptable conduct.

Objectives

The current study has the following specific objectives:

1. To examine the different dimensions of the surveillance system for TB in Afghanistan.

2. To evaluate the level of PHC facilities involvement in TB active case findings per BPHS requirements.

\section{Result}


Sensitivity is the ability of information and surveillance system to detect cases and epidemics timely. In a case-based surveillance system, it refers to the proportion of cases in a population that is notified through the surveillance system. To calculate sensitivity, it is essential to divide the number of reported confirmed cases by the number of cases in the community (ECDC, 2014).

The overall sensitivity of TB surveillance systems in the country counts to $56.30 \%$. This means that $43.70 \%$ of TB cases had remained undetected. Khost and Bamyan provinces both have the highest and lowest TB surveillance sensitivity and they each account to $101.61 \%$ and $29.92 \%$ respectively.

Badakhshan with $33.28 \%$ is the second-lowest. The results for Nangarhar, Kunar, Kandahar, Herat, Balkh, Herat, and Kabul were 87.81, 77.27, 62.97, 49.70, 48.12, and 42.08\% respectively. Table-2 indicates the sensitivity of surveillance systems to detect TB cases by province from August to November 2016.

The scale of active case findings in primary health care facilities

In the light of global urgency to improve TB case detection to curb TB epidemics, innovative active case findings strategies have invited enormous attention. In studied health facilities, $43.81 \%$ used at least one accepted active case finding strategies while $56.20 \%$ implemented none. Figure- 1 highlights the facilities with one active case finding strategies and facilities which had no strategy.

Among facilities that reported having any active case finding interventions, $17 \%$ conducted contact screening, $9.92 \%$ implemented health education, $6.61 \%$ required $\mathrm{CHWs}$ to actively look for TB suspect cases in the community and $5.79 \%$ employed a mix of health education, triage, and contract screening. The figure-2 highlights the different case-finding strategies:

The availability of data on the number of TB cases in the catchment areas within health facilities helps the health facilities management team undertake outreach activities, identify the population at risk, and accelerate targeted preventive interventions. From studied BHCs $27 \%$ had information on the number of TB cases in their catchment areas. $8.33 . \%$ had some information but $64.54 \%$ had no information. In $\mathrm{CHCs}, 61.54$ had information, $7.69 \%$ had relative information, and $30.77 \%$ had no information. In DHs, $58.33 \%$ had information, 41.67 had no information. Figure -3 demonstrates the availability of information to TB cases in catchment areas of health facilities.

\section{Discussion}

The study found that the overall sensitivity of TB surveillance systems in-country is $56.30 \%$. This means that $43.70 \%$ of TB cases had remained undetected. Unnoticed cases not only spread infection but are susceptible to severity and resistance. These findings are similar to global TB report 2015 and World Bank data as they show the same patterns. Mentioned report and the data confirmed that in 2015 only $55 \%$ of cases were detected.

As study implemented in 2014, claimed that improving the TB case detection has remained a big struggle since the last decade. It adds that only half of the projected incident TB cases were reported and the 
notifications have reduced since the top time in 2007 (Sanaie, 2016). Poor system sensitivity is not only a problem in Afghanistan but it is also a severe challenge in the neighboring countries. For example, a study of TB surveillance system in Sindh province in Pakistan identified the sensitivity at $20 \%$ (Asif, 2012).

$43.81 \%$ of studied facilities used at least one accepted active case finding strategy while $56.20 \%$ implemented none. The widespread active case findings strategies in developing countries embrace contact investigation, targeted testing of high-risk populations, and outbreak investigations. TB high-risk population comprises immigrants, refugees, internally displaced people, and addicts (CDC, 2016).

This study measured the passive case finding at $72 \%$. The studied facilities were sending all clients with longer than 2 weeks of couth for further checkups to upper-level facilities. $57 \%$ of studied facilities conducted further checkups when patients had a cough of any duration, hemoptysis, weight loss, fever, and nightly sweats. These obtained values are significantly lower $(0.014$ and 0.0001$)$ than intended.

After the clients are referred by health posts, sub-centers, and basic health centers to upper-level facilities, most of them either don't visit these upper-level facilities or visit these upper-level facilities but don't return to the initial centers from where they were referred. The health posts, sub-centers, and basic health centers besides referral role, should also have a role in patients' education, motivation, and direct observation therapy, and strategies need to be implemented to ensure clients come back to initial facilities to start and complete their treatment.

A study conducted in 2016, examined the effectiveness of three case fining strategies in six provinces of Afghanistan. The active case finding strategies comprised systematic screening of health facilities' attendees for cough, active contact tracing of smear-positive index TB patients, and active screenings among the residents of internally displaced people's camps. The study determined that while most patients with TB are likely to be identified through health facility screening but many people remain without a proper diagnosis if these mentioned three active case finding strategies were not implemented (Sanaie, 2016).

\section{Recommendations}

To improve the sensitivity of TB surveillance system, it is essential to strengthen passive case finding and conduct active case finding (communicating with doctors at hospitals, private clinics, and labs). Based on experience and reviewed literature, the following interventions are suggested to boost sensitivity and increase cases detection rate:

1. Undertake case-finding strategies among high-risk populations in different areas. The high-risk groups include IDPs, returnees, slum residents, prisoners and addicts

2. Training the regional and provincial TB coordinators and community influencers to support community-based interventions for TB cases finding. 
3. Involve private pharmacies and traditional healers to identify and refer individuals with TB symptoms for follow up and further investigation in public facilities.

4. Assign trained sputum fixers who collect sputum from symptomatic patients at the community level or remote health facilities which don't have smear microscopic equipment and deliver the slides to nearest DOTs facilities.

\section{Abbreviations}

Basic Health Center (BHC), 4

Comprehensive Health Center (CHC), 4

Hospital (DH), 4

Management Information System (HMIS), 3

Mobile Health Team (MHT), 4

Sub Health Center (SHC, 4

Tuberculosis (TB), 3

World Health Organization (WHO), 3

Direct Observation Therapy (DOTs), 9;

Institutional Review Board (IRB), 5

\section{Declarations}

\section{Ethics approval and consent to participate}

The study received ethical approval from MoPH's Institutional Review Board (IRB). All participants provided informed consent before participating in the study. For patients, the interviewers read the informed consent and described the objectives, benefits, voluntary participation, confidentiality, and rights of respondents' withdrawal. The enumerators had also got trained on local cultural norms and socially acceptable conduct.

Consent for publication

NA

Availability of data and materials 
The datasets used and/or analyzed during the current study are available from the corresponding author on reasonable request.

\section{Competing interests}

The authors declare that they have no competing interests.

\section{Funding}

UNDP from their GF grant financed this study.

\section{Acknowledgments}

We acknowledge the contribution of the NTP technical team, UNDP TB project team, Implementing NGOs field teams, and health facilities staff.

\section{Authors' contributions}

AA provided feedback on the study to improve the write-up. KS provides contributed to the analysis and field data collection supervision. All authors read and approved the final manuscript.

\section{References}

1. Aloudal. (2015). ReliefWeb. Retrieved from Report:

http://reliefweb.int/report/afghanistan/tuberculosis-kills-13-000-afghans-every-year.

2. Asif M. (2012). Evaluation of the Tuberculosis Surveillance System in District Hyderabad, Province Sindh-Pakistan. TROPICAL DISEASE \& Health. Retrieved from http://www.sciencedomain.org/abstract/9476.

3. CDC. (2016). Guidelines for Evaluating Surveillance Systems. Department of Health and Humen Services. Retrieved 2017, from https://www.cdc.gov/mmwr/preview/mmwrhtml/00001769.htm.

4. CDC. Targeted Tuberculosis (TB) Testing and. Atlanta: CDC; 2016.

5. ECDC. (2014). Data quality monitoring and surveillance system evaluation. Stockholm: European Centre for Disease Prevention and Control. Retrieved from http://ecdc.europa.eu/en/publications/publications/data-quality-monitoring-surveillance-systemevaluation-sept-2014.pdf.

6. Ernst, Young. Micro Assessment. Kabul: UNDP; 2015.

7. Sanaie. (2016). An Evaluation of Passive and Active Approaches to Improve Tuberculosis Notifications in Afghanistan. PLoS One. Retrieved from https://www.ncbi.nlm.nih.gov/pubmed/27701446. 
8. Seddiq. (2014). Implementing a successful tuberculosis program within primary care services in a conflict area using the stop TB strategy: Afghanistan case study. Conflict and Health, 06.

9. WHO. A guide to understanding the TB control strategy known as DOTS. Geneva: WHO; 2000.

10. WHO

WHO. (2010). WHO. Retrieved from Stop TB. Strategy:

http://www.who.int/tb/strategy/stop_tb_strategy/en/.

11. WHO. PLANNING INTEGRATED HIV SERVICES AT THE HEALTH CENTR. Geneva: WHO; 2014.

12. WHO. The Urban Health Index: A handbook for its calculation and use. Japan: Kobe; 2014.

13. WHO

WHO. (2015). WHO. Retrieved from End TB. strategy:

http://www.who.int/tb/strategy/End_TB_Strategy.pdf?ua=1.

14. WHO. (2016). Tuberculosis Global Report. Geneva: World Health Organization. Retrieved 2017, from http://www.who.int/tb/publications/global_report/gtbr2016_executive_summary.pdf?ua=1.

15. WHO. (2017). WHO. Retrieved from Sustainable Development Goals:

http://www.who.int/topics/sustainable-development-goals/targets/en/.

\section{Figures}

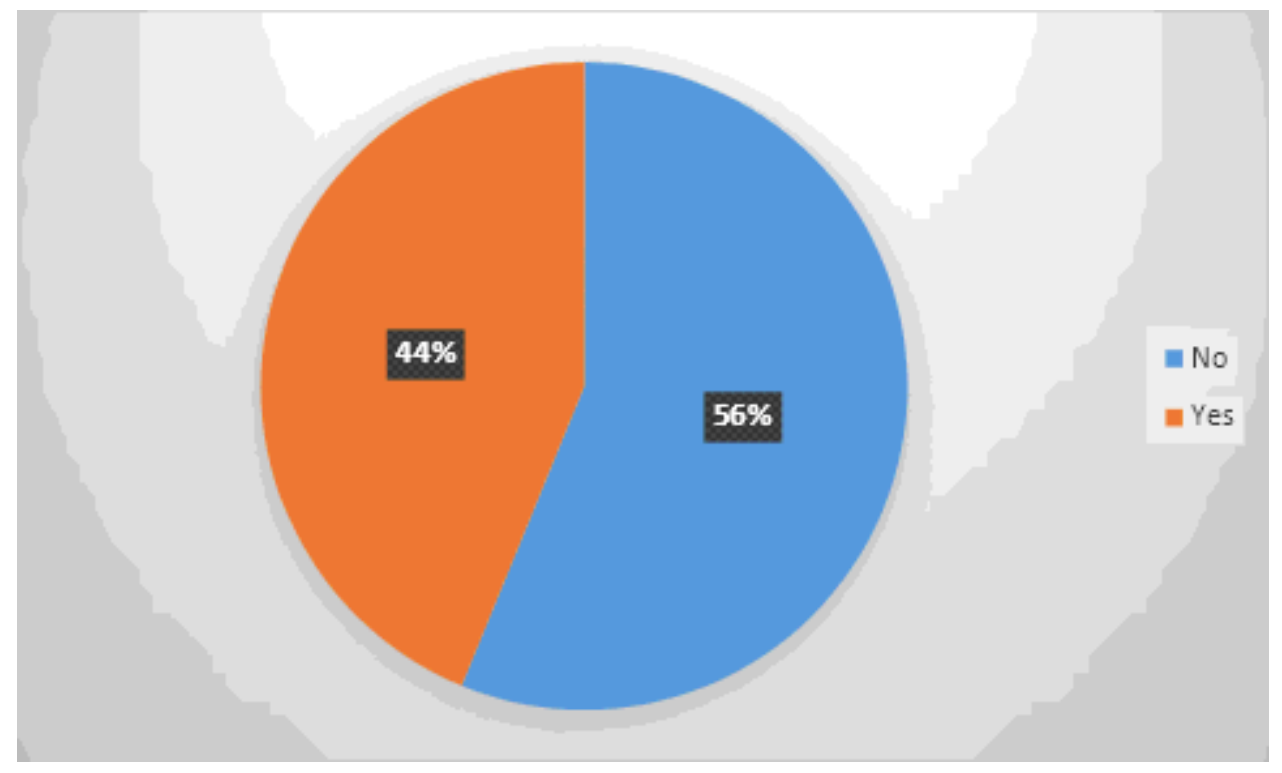

\section{Figure 1}

Case finding strategies in health facilities 


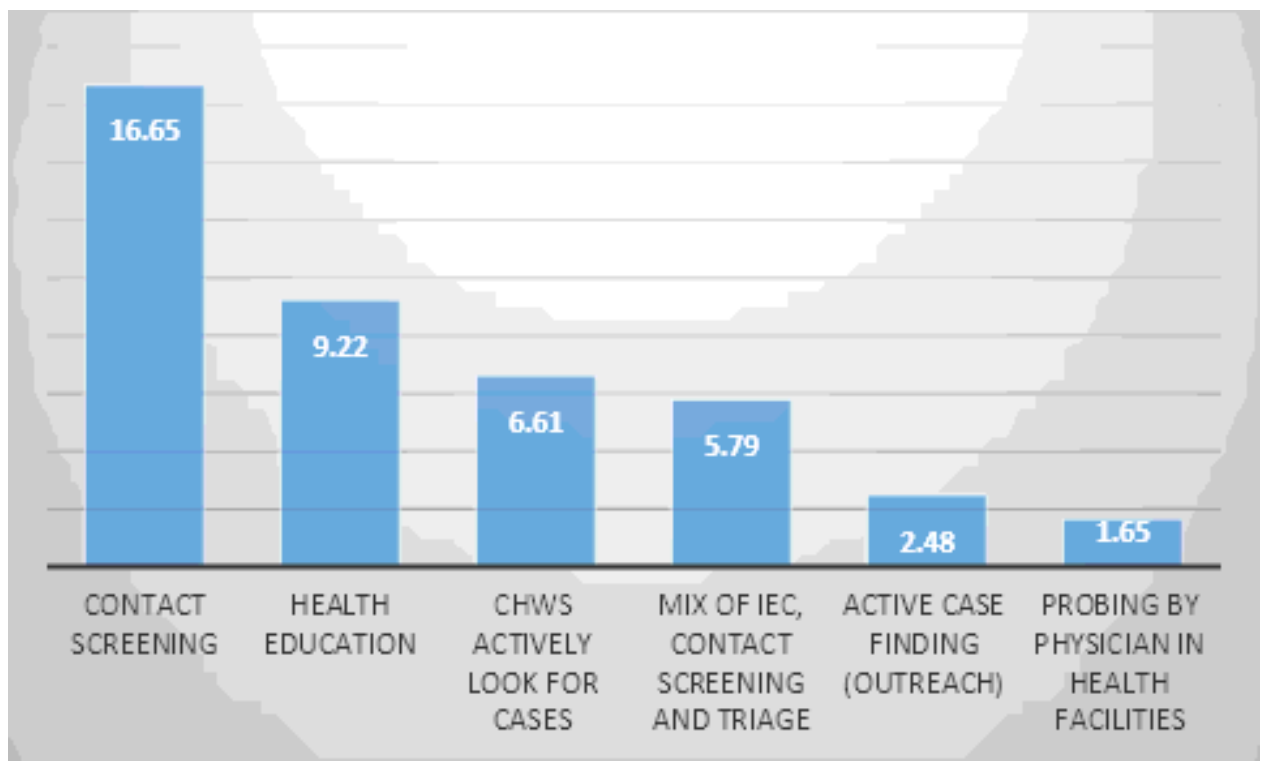

Figure 2

Types of active case finding strategies

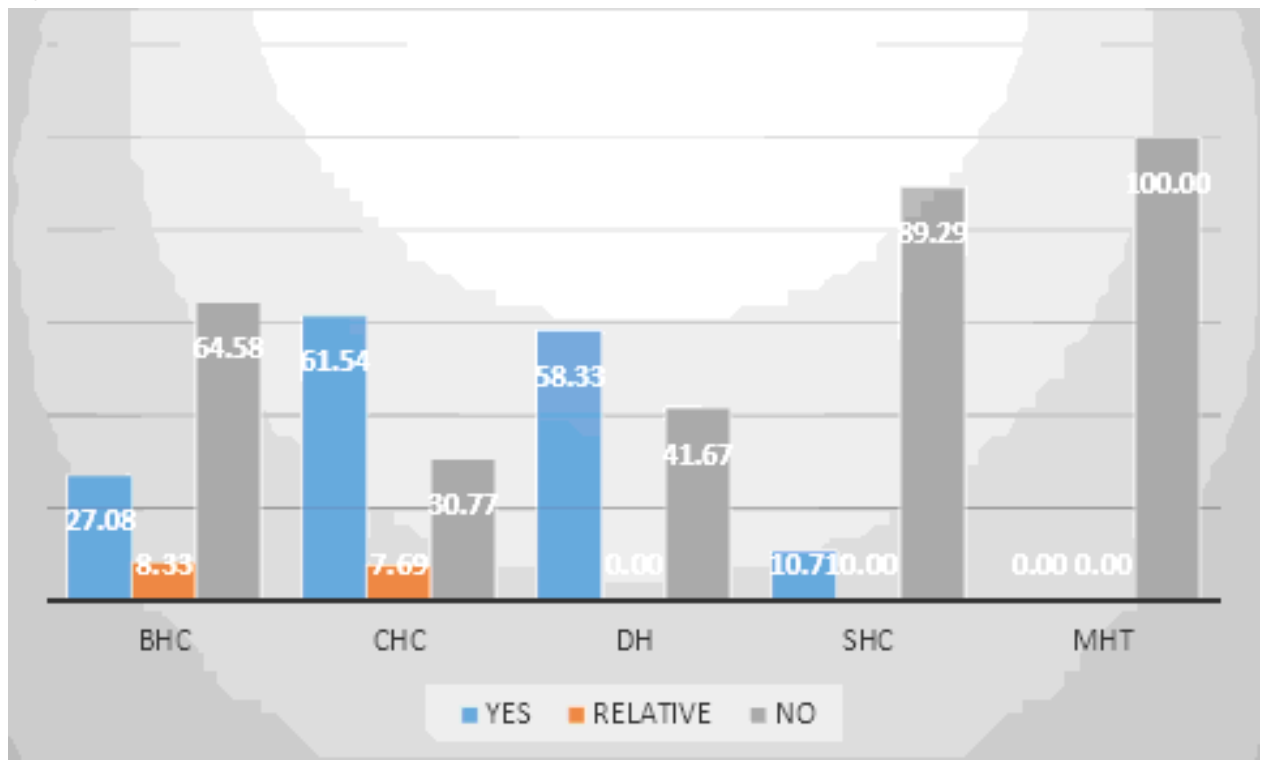

Figure 3

Available information on the number of TB cases in HF catchment areas 